\title{
Quantitative Methods for Testing Antiviral Activities of Textile Fabrics
}

\author{
Yasuo Imoto ${ }^{1,2^{*}}$, Satoshi Seino ${ }^{2}$, Takashi Nakagawa ${ }^{2}$ and Takao A Yamamoto ${ }^{2}$ \\ ${ }^{1}$ Japan Textile Products Quality and Technology Center, 5-7-3, Shimoyamate-dori, Chuo-ku, Kobe-city, Hyogo, Japan \\ ${ }^{2}$ Graduate School of Engineering, Osaka University, 2-1 Yamadaoka, Suita, Osaka, Japan
}

*Corresponding author: Yasuo Imoto, Japan Textile Products Quality and Technology Center, 5-7-3, Shimoyamate-dori, Chuo-ku, Kobe-city, Hyogo, 650-0011, Japan, Tel: 81-78-351-1909; Fax: 81-78-351-1901; E-mail: y-imoto@qtec.or.jp

Received date: June 14, 2017; Accepted date: July 18, 2017; Published date: July 25, 2017

Copyright: (c) 2017 Imoto Y, et al. This is an open-access article distributed under the terms of the Creative Commons Attribution License, which permits unrestricted use, distribution, and reproduction in any medium, provided the original author and source are credited.

\begin{abstract}
Objective: In recent years, antiviral products modified with various antiviral agents are now widely studied and developed. The establishment of appropriate antiviral testing methods for such products is necessary to discuss the efficiency of antiviral agents in the products. In this study, quantitative antiviral testing methods for textile fabrics were studied under several test conditions.

Method: Influenza virus and feline calicivirus were used as model viruses. A cotton fabric and a $100 \%$ polyester fabric were used as control samples and $\mathrm{Ag}$ nanoparticles were used as model antiviral agents. In testing the antiviral performance of a product with an antiviral treatment, a virus suspension is inoculated onto the product and subsequently washed out of the products. The virus infectivity titer in the washed-out suspension is then measured. The viral infectivity titer of the test suspension was determined by counting the plaque-forming units (PFU). In estimating the antiviral activity of textile products, the components of the virus suspension, the contact time, and the contact temperature were chosen as study points.
\end{abstract}

Results: The virus infectivity titer was significantly affected by experimental conditions such as the concentration of the medium of the virus suspension, the contact temperature, and the type of textile fabric support. To estimate the antiviral efficiency under stable conditions for viruses, a lower temperature and a lower Eagle's minimal essential medium (EMEM) concentration appear to be appropriate. It was confirmed that Ag nanoparticles on textile fabrics have antiviral activity; however, this antiviral activity was suppressed in media of high concentrations.

Conclusions: It was shown that inactivation of antiviral agent might be caused by components of the virus suspension, as well as by other experimental conditions. Furthermore, the viruses can be deactivated by contact with textile fabrics, even in the absence of antiviral agents. These findings indicate that the antiviral performance testing conditions must be carefully chosen with due consideration of the field of application.

Keywords: Antiviral activity; Influenza A virus; Feline calicivirus; Ag nanoparticles; Virus suspension; Antiviral efficiency; Eagle’s minimal essential medium

\section{Introduction}

In recent years, demand for antiviral products has been expanding in many fields where the aim is to achieve a comfortable and clean life. Furthermore, there has also been an increase in interest in infections by such viruses as the influenza virus, norovirus, and respiratory syncytial virus. Various antiviral products have been developed in response to these circumstances, and various types of antiviral agent for use in such products are now widely studied and developed. However, their antiviral mechanisms have not been thoroughly investigated. As a result of these factors, the establishment of appropriate antiviral testing methods is necessary from the view-points of both basic science and applications.

There are many reports, with many study examples, on evaluation methods for products with antimicrobial finishes. Bacteria and molds have the ability to self-propagate through metabolism under nutrient conditions. Consequently, most evaluation systems are designed to evaluate the inhibitory effect of treatment agents on the proliferation of bacteria and molds by incubating these microorganisms on the surfaces a test subjects or inside the subjects [1-4]. Because viruses do not have a self-multiplication mechanism, these quantitative analysis strategies cannot be applied in testing antiviral products [5]. Antiviral activity therefore needs to be evaluated from the changes in the virus infectivity titer resulting from contact between a virus suspension and an antiviral finishing product. However, such methods have yet to be firmly established.

To establish a testing method, the following points should be considered. First, even when antiviral agents are not present under a set of test conditions, it necessary to take into account the possibility of virus inactivation as a result of, for example, concentrations of coexisting materials or the contact temperature. A test virus suspension is prepared by multiplying viruses infected into host cells. Eagle's minimal essential medium (EMEM) is generally used in multiplying an influenza virus or feline calicivirus by cell culture [6,7]. Although EMEM is an optimized growth medium for culturing host cells, many of its components, such as amino acids, inorganic salts, and vitamins, might operate as inactivation factors for viruses. Secondly, it is necessary to take into account the possibility of virus inactivation by components other than antiviral agents on the surfaces of products lacking an antiviral treatment. In testing the antiviral performance of a 
product with an antiviral treatment, a virus suspension is inoculated onto the product and subsequently washed out of the products. The virus infectivity titer in the washed-out suspension is then measured. It is therefore necessary to take into account both the virus washout efficiency from the product and virus inactivation by contact with the surface of product. In the case of the quantitative evaluation of fungal activities on textile fabrics, the fungal growth activity values obtained by means of the colony-counting method depend on the morphological state of the fungi on the fabric, because the morphological state affects the wash-out efficiency of fungi from textile fabrics [8]. Although viruses do not undergo morphological transformations on textile fabrics, it is necessary to make allowances for the wash-out efficiency of viruses from these materials.

In this study, an antiviral performance test was studied by using textile products as examples of anti-virally treated products. First, we investigated whether either the concentration of EMEM used in preparing the virus suspension or the temperature at which the test was conducted affected the virus infectivity titer. Then, by using untreated textiles, we investigated the virus washout efficiency of the textiles and virus inactivation by contact with the textiles. Finally, we investigated the antiviral activity of an anti-virally treated textile prepared by immobilizing silver nanoparticles on cotton fabric.

\section{Materials and Methods}

In estimating the antiviral activity of textile products, the components of the virus suspension, the contact time, and the contact temperature were chosen as study points. We evaluated the change over time of the virus infectivity titer on a $100 \%$ cotton fabric, without the fluorescent brighteners or other finishes mentioned in ISO 18184 [8], and on a $100 \%$ polyester fabric. These were treated with a virus suspension, and any differences in the efficiency of wash-out of the virus from the textile products caused by the textile materials were analyzed. Additionally, we evaluated the changes over time in the virus infectivity titer caused by the components of the test virus suspension, by the contact time, and by the contact temperature, and we determined the optimal test conditions for the antiviral test. Initially, we chose a contact time of 2-24 hours, and contact temperatures of $4^{\circ} \mathrm{C}$, because the virus is stable at this temperature, and $25^{\circ} \mathrm{C}$, because of the presumed environment for the use of the antivirally finished textile products. Experiments are repeated 3 times per 1 experimental data, and the average value of 3 experimental data is adopted.

Treatment of virus suspensions by ultrafiltration or ultracentrifugation or purification by the density-gradient method are effective methods for suppressing the effects of cell debris in the virus suspension or EMEM on the virus infectivity titer. In this study, however, we chose the method of dilution with purified water, which reduces the effect of the EMEM on the virus infectivity titer, and does not need any specific operations.

\section{Preparation of test specimens}

A $100 \%$ cotton fabric, without fluorescent brighteners or other finish mentioned in ISO 18184 [9], and a 100\% polyester fabric were used as control samples. An antiviral textile fabric was prepared by immobilizing Ag nanoparticles onto the cotton fabric by using a radiochemical technique reported elsewhere [8]. The amount of Ag nanoparticles on the fabric was controlled by the precursor to give 146 $\mu \mathrm{g}$ of Ag per gram of fiber. Specimens with a mass of 0.4 grams were each placed in a $30 \mathrm{ml}$ vial that was autoclaved at $121^{\circ} \mathrm{C}$ for 15 minutes. After sterilization, the vials were transferred to a safety cabinet for 60 minutes to cool and dry the specimens.

\section{Test virus}

We used two viral species and two host cell lines, as described in ISO 18184 [9]. The two viral species were Influenza A virus (H3N2) (A/Hong Kong/8/68; TC adapted ATCC VR-1678), an enveloped virus, and feline calicivirus strain F-9 ATCC VR-782, a non-enveloped virus. The host cell lines were MDCK cells ATCC CCL-34 for the Influenza A virus and CRFK cells; ATCC CCL-94 for the feline calicivirus.

\section{Preparation of virus suspensions}

The viral suspensions were prepared according to ISO 18184 [9].

Influenza virus: The growth medium was drained from a $75 \mathrm{ml}$ flask containing MDCK cells cultured in a monolayer at $37^{\circ} \mathrm{C}$ under $5 \%$ $\mathrm{CO}_{2}$ for three days in EMEM containing $10 \%$ fetal bovine serum (FBS). The surface of the cultured cells was washed with EMEM, and the medium was drained. This washing procedure was repeated twice. A suspension of influenza virus $(1.0 \mathrm{ml})$, prepared to a concentration of approximately $10^{3} \mathrm{PFU} / \mathrm{ml}$, was inoculated onto the surface of the cells in the flask, and spread over the entire surface. The flask was placed in a $\mathrm{CO}_{2}$ incubator at $34^{\circ} \mathrm{C}$ and kept at this temperature for one hour to adsorb the virus onto the cells. EMEM containing $1.5 \mathrm{ppm}$ trypsin derived from beef pancreas was added to the flask, and the preparation was incubated in the $\mathrm{CO}_{2}$ incubator at $34^{\circ} \mathrm{C}$ for two days to multiply the influenza virus. The multiplied virus suspension was centrifuged at $4^{\circ} \mathrm{C}$ and 1000 grams for 15 minutes. The supernatant was then removed from the centrifugation tube. The concentration of the resulting virus suspension was approximately $10^{8} \mathrm{PFU} / \mathrm{ml}$. Finally, the virus suspension was diluted tenfold with EMEM or purified water as a diluent to give a test suspension of the influenza virus with a concentration of approximately $10^{7} \mathrm{PFU} / \mathrm{ml}$. The virus suspension diluted by EMEM is referred to henceforth as the 1/1 EMEM virus suspension, and that diluted by purified water is referred to as the $1 / 10$ EMEM virus suspension.

Feline calicivirus: Growth medium was drained from a $75 \mathrm{ml}$ flask containing CRFK cells cultured in a monolayer at $37^{\circ} \mathrm{C}$ under $5 \% \mathrm{CO}_{2}$ for three days in Dulbecco's modified Eagle's medium (DMEM) containing $10 \%$ FBS. The surface of the cultured cells was washed with EMEM, and the medium was drained. The washing procedure was repeated twice. A suspension of feline calicivirus $(1.0 \mathrm{ml})$ prepared to a concentration of approximately $10^{7} \mathrm{PFU} / \mathrm{ml}$ was inoculated onto the surface of the cells in the flask and was spread over the entire surface. The flask was placed in a $\mathrm{CO}_{2}$ incubator at $37^{\circ} \mathrm{C}$ and kept at this temperature for one hour to adsorb the virus onto the cells. EMEM was added, and the flask was incubated in the $\mathrm{CO}_{2}$ incubator at $37^{\circ} \mathrm{C}$ for one day to multiply the feline calicivirus. The multiplied virus suspension was centrifuged at $4^{\circ} \mathrm{C}$ and 1000 grams for 15 minutes. The supernatant was then removed from the centrifugation tube. The concentration of the resulting virus suspension was approximately $10^{8}$ $\mathrm{PFU} / \mathrm{ml}$. Finally, this virus suspension was diluted tenfold with EMEM or purified water as diluents to give a test suspension of feline calicivirus with a concentration of approximately $10^{7} \mathrm{PFU} / \mathrm{ml}$. The virus suspension diluted by EMEM is referred to hereafter as the $1 / 1$ EMEM virus suspension, and that diluted by purified water is referred to as the 1/10 EMEM virus suspension. 


\section{Inoculation and plaque-counting method}

The virus infectivity titer was measured by a plaque-counting method performed according to the ISO 18184 guideline [9]. Each specimen was inoculated with $0.2 \mathrm{ml}$ of virus suspension $\left(1.0 \times 10^{7}\right.$ $\mathrm{PFU} / \mathrm{ml})$. A $50 \mathrm{ml}$ centrifugation tube was charged with $0.2 \mathrm{ml}$ of the virus suspension $\left(1.0 \times 10^{7} \mathrm{PFU} / \mathrm{ml}\right)$ to analyze the stability of the virus in a virus-only suspension. After inoculation, the specimens were kept at $4^{\circ} \mathrm{C}$ or $25^{\circ} \mathrm{C}$ for 2,12 , or 24 hours. Subsequently, $20 \mathrm{ml}$ of soybean casein lecithin polysorbate 80 (SCDLP) medium was added to each vial or centrifugation tube, and the viruses were washed out by vortexing five times for five seconds each time.

The virus infectivity titer in the washed-out suspension was determined by enumerating plaque-forming units (PFU).

\section{Results and Discussion}

\section{Temporal changes in virus infectivity titers}

Figure 1a shows the changes in the feline calicivirus infectivity titer over time as functions of the components of the virus suspension and the contact time. When the contact temperature was $4^{\circ} \mathrm{C}$, the infectivity titer of the feline calicivirus in both the 1/1 EMEM virus suspension and the 1/10 EMEM virus suspension showed only a slight decrease. The infectivity titer of the feline calicivirus in the 1/10 EMEM virus suspension at $25^{\circ} \mathrm{C}$ also showed only a slight decrease, whereas the infectivity titer of the feline calicivirus in the 1/1 EMEM virus suspension at $25^{\circ} \mathrm{C}$ showed a significant decrease.

Figure $1 \mathrm{~b}$ shows the changes in the influenza virus infectivity titer over time as functions of the composition of the virus suspension and the contact time. The infectivity titer of the influenza virus in the $1 / 10$ EMEM virus suspension at $4^{\circ} \mathrm{C}$ showed only a slight decrease whereas the infectivity titer of the influenza virus in the 1/1 EMEM virus suspension at $4^{\circ} \mathrm{C}$ showed a small decrease after a 24 hour contact period. The infectivity titer of the influenza virus in the 1/1 EMEM virus suspension at $25^{\circ} \mathrm{C}$ showed a single-digit decrease after a 24 hour contact period. The infectivity titer of the influenza virus in the $1 / 10$ EMEM virus suspension at $25^{\circ} \mathrm{C}$ showed little decrease after a 24 hour contact period.

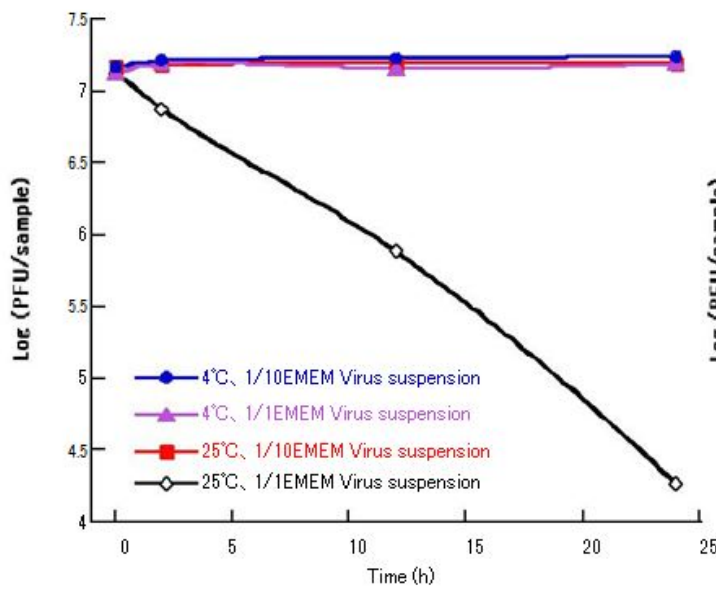

(a)

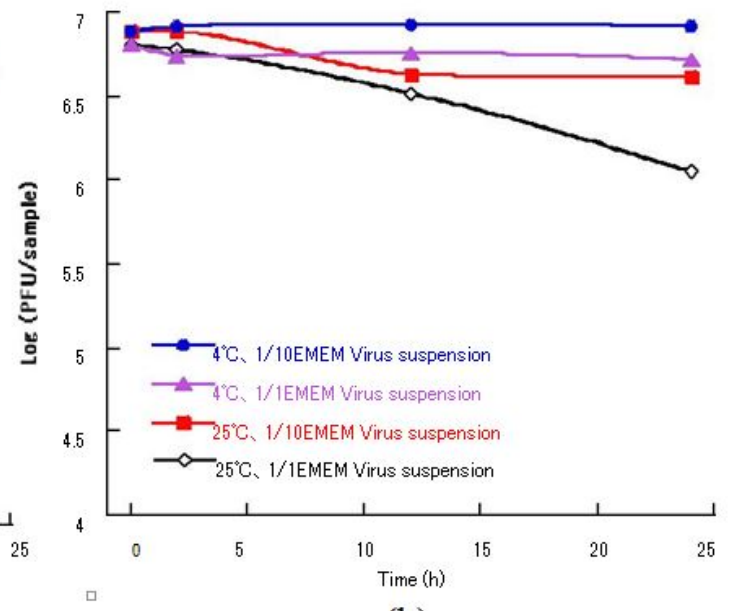

(b)

Figure 1: Changes in the virus infectivity titer in virus suspensions: (a) feline calicivirus; (b) influenza A virus.

We therefore showed that the experimental conditions of the EMEM concentration and the contact temperature can affect virus inactivation. As already mentioned, EMEM is widely used as a medium for test virus suspensions because it is an optimized growth medium for culturing host cells. Our results show that the concentration of EMEM and the contact temperatures must be carefully chosen to construct appropriate conditions for testing virus inactivation. We also showed that inactivation behaviour depends on the type of virus; this difference might result from the presence or absence of a viral envelope.

\section{Changes in virus infectivity titer on textiles}

To evaluate the activity of antiviral agent immobilized on textile fabrics, the virus suspension was first dropped onto the textile fabric. After the viruses had been in contact with the antiviral agent for a specified time, the viruses were washed, and the viral infectivity titer was determined by PFU testing. It was necessary, therefore, to take into account the adsorption of the virus by the fiber material and/or the virus inactivation caused by the fiber material itself. In this study, the adsorption and/or inactivation of viruses by the textile fabrics without antiviral agents were investigated by using a $100 \%$ cotton fabric and $100 \%$ polyester fabric.

Figure 2 shows the changes in the feline calicivirus infectivity titer over time as a function of the nature of the untreated textile fabric, the composition of the virus suspension, and the contact temperature. When the contact temperature was $4^{\circ} \mathrm{C}$ and virus suspension was the 1/10 EMEM virus suspension (Figure 2a), the feline calicivirus infectivity titer in the presence or absence of the textile fabric was more or less the same. These results showed that the virus was washed out from the textile fabrics efficiently under the test conditions, and that virus was not inactivated by the fiber materials. Under other experimental conditions (Figures $2 \mathrm{~b}-2 \mathrm{~d}$ ), the infectivity titer of the 
feline calicivirus in the presence of the textile fabrics showed slight decrements compared with the titers in the absence of the textile fabrics, probably due to adsorption and/or inactivation by the textile fabrics. Although the decrement was relatively small, this adsorption and/or inactivation must be taken into account when performing antiviral tests under these experimental conditions.
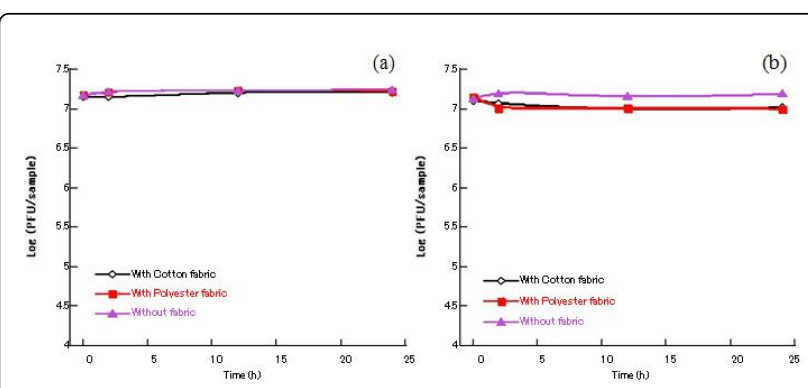

(c)

(d)
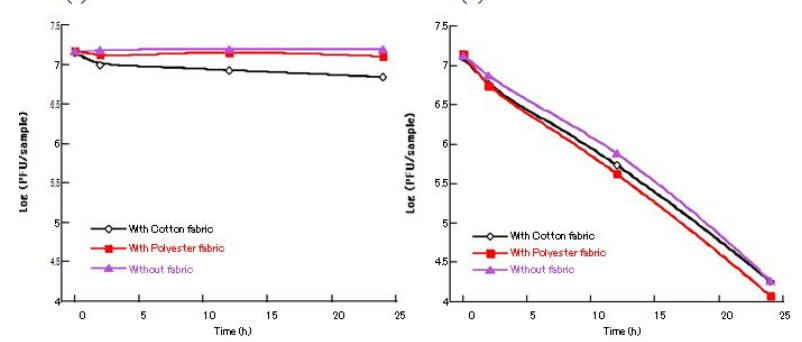

Figure 2: Changes in the feline calicivirus infectivity titer: (a) $4^{\circ} \mathrm{C}$, $1 / 10$ EMEM virus suspension; (b) $4^{\circ} \mathrm{C}, 1 / 1$ EMEM virus suspension; (c) $25^{\circ} \mathrm{C}, 1 / 10 \mathrm{EMEM}$ virus suspension; (d) $25^{\circ} \mathrm{C}, 1 / 1$ EMEM virus suspension.
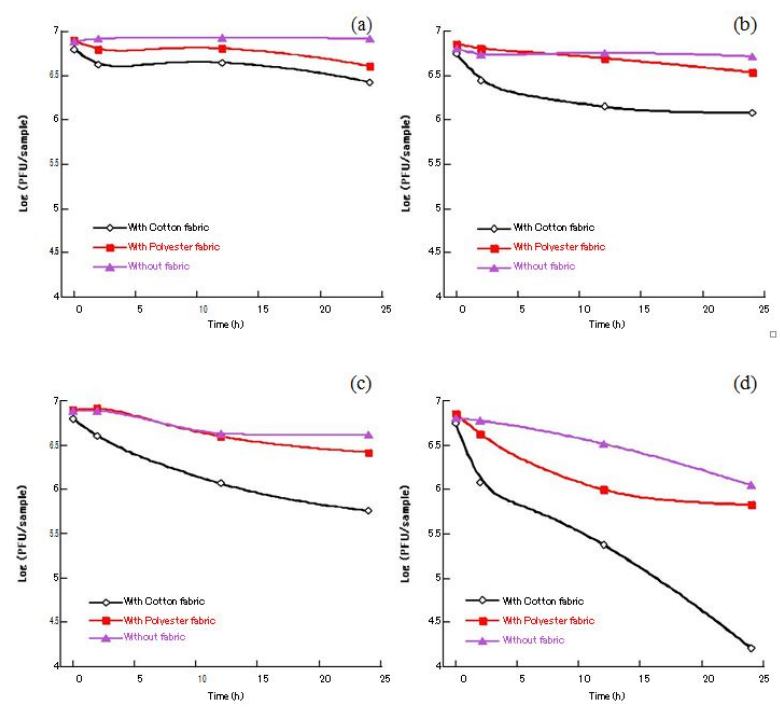

(c)

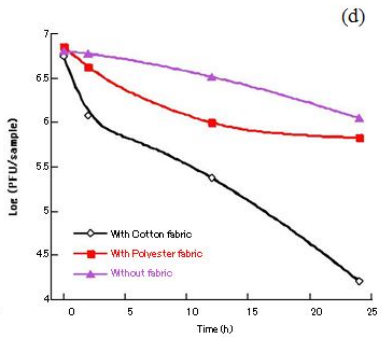

Figure 3: Changes in the influenza virus infectivity titer: (a) $4^{\circ} \mathrm{C}$, $1 / 10$ EMEM virus suspension; (b) $4^{\circ} \mathrm{C}, 1 / 1 \mathrm{EMEM}$ virus suspension; (c) $25^{\circ} \mathrm{C}, 1 / 10$ EMEM virus suspension; (d) $25^{\circ} \mathrm{C}, 1 / 1$ EMEM virus suspension.

Figure 3 shows the changes in the influenza virus infectivity titer over time as a function of the type of untreated textile fabrics, the composition of virus suspension and contact temperature. Compared with the corresponding results for the feline calicivirus (Figure 2), the infectivity titer of the influenza virus showed a significant decrease as a result of contact with the textile fabric, especially the cotton fabric. These results indicate that the washing out efficiency and/or degree of inactivation of viruses depends on the experimental conditions (contacting time, temperature, and EMEM concentration), the type of textile fabric, and the nature of the test virus. Therefore, in antiviral performance tests using anti-virally treated textile products, a relative comparison with untreated specimens is essential. Furthermore, it is also necessary to choose appropriate test conditions for each specific characteristic of the test product. Among the present experimental conditions, a lower temperature, a shorter contact time, and a low EMEM concentration appear to be appropriate.

We have therefore shown that the influenza virus showed a more significant decrement in infectivity titer on contact with the textile fabrics than did the feline calicivirus. At the present stage, it is difficult to determine whether this decrement is caused by adsorption or inactivation, and further study is required.

\section{Effects of silver treatment of fabrics}

As shown above, in antiviral performance testing of anti-virally treated textile products, a comparison with untreated specimens is essential when examining the virus inactivation caused by the treated textile material. We therefore conducted antiviral tests by using Agtreated cotton fabric and untreated cotton fabric with the 1/1 EMEM virus suspension and the 1/10 EMEM virus suspension. The contact temperature was $25^{\circ} \mathrm{C}$.

\begin{tabular}{|l|l|l|l|l|l|}
\hline \multirow{4}{*}{ Virus } & \multirow{2}{*}{$\begin{array}{l}\text { EMEM } \\
\text { conc. }\end{array}$} & \multirow{2}{*}{ Textile } & \multicolumn{3}{|l|}{ Contact Time (hour) } \\
\cline { 4 - 7 } & & & $\mathbf{0}$ & $\mathbf{2}$ & $\mathbf{1 8}$ \\
\hline \multirow{4}{*}{$\begin{array}{l}\text { Feline } \\
\text { calicivirus }\end{array}$} & 01-Oct & no Ag & 6.91 & 6.81 & 6.34 \\
\cline { 2 - 7 } & $01-$ Oct & with Ag & 6.89 & 6.2 & 2.3 \\
\cline { 2 - 7 } & 01-Jan & no Ag & 6.92 & 6.43 & 2.78 \\
\cline { 2 - 7 } & 01-Jan & with Ag & 6.89 & 6.06 & 3.08 \\
\hline \multirow{4}{*}{$\begin{array}{l}\text { Influenza } \\
\text { virus }\end{array}$} & 01-Oct & no Ag & 6.71 & 6.43 & - \\
\cline { 2 - 7 } & 01-Oct & with Ag & 6.67 & 2.3 & - \\
\cline { 2 - 7 } & 01-Jan & no Ag & 7.11 & 6.74 & - \\
\cline { 2 - 7 } & 01-Jan & with Ag & 7.07 & 6.15 & - \\
\hline
\end{tabular}

Table 1: Changes in the virus infectivity titer over time on cotton fabrics.

Table 1 shows the changes in the virus infectivity titer over time on Ag-treated cotton fabric. There was significant difference in the feline calicivirus infectivity titer in the 1/10 EMEM virus suspension between the Ag-treated and the untreated cotton fabrics. On the other hand, there was no difference in the feline calicivirus infectivity titer in the 1/1 EMEM virus suspension between the Ag-treated and the untreated cotton fabrics. The feline calicivirus infectivity titer in 1/1 EMEM virus suspension at $25^{\circ} \mathrm{C}$, as mentioned above, decreased over time on both Ag-treated and untreated cotton fabrics. Because the feline calicivirus infectivity titer showed a significant decrease on untreated cotton fabric, the antiviral effect of Ag treatment could not be estimated. On 
the other hand, the feline calicivirus infectivity titer in 1/10 EMEM virus suspension at $25^{\circ} \mathrm{C}$ showed a significant decrease on the Agtreated cotton fabric after an 18 hour contact period. Under these conditions, the feline calicivirus infectivity titer in the 1/10 EMEM virus suspension at $25^{\circ} \mathrm{C}$ showed a slight decrease on the untreated cotton fabric. Therefore, a significant antiviral effect of Ag treatment was demonstrated after 18 hours of contact.

The influenza virus infectivity titer in the 1/10 EMEM virus suspension at $25^{\circ} \mathrm{C}$ showed a significant decrease on the Ag-treated cotton fabric after a two hour contact period. On the other hand, the influenza virus infectivity titer in the $1 / 1$ EMEM virus suspension at $25^{\circ} \mathrm{C}$ showed only a slight decrease on the Ag-treated cotton fabric after a two hour contact period. These results showed that the concentration of EMEM might be a factor in the inactivation of the antiviral effect of the Ag nanoparticles. The reason for the inactivation of the Ag nanoparticles can be discussed by using their antibacterial mechanism as a reference. The antibacterial mechanism of $\mathrm{Ag}$ nanoparticles is usually discussed on the basis of their surface activity $[10,11]$. It has also been reported that the surface of $\mathrm{Ag}$ nanoparticle can be easily passivated by thiol ( $\mathrm{SH}$ ) groups, resulting in a loss of antibacterial activity [12]. Because EMEM contain cysteine, an amino acid with a thiol group in its structure, surface passivation of the Ag nanoparticles might proceed before contact with the viruses. Although the antiviral mechanism of $\mathrm{Ag}$ nanoparticles is not understood, this surface passivation might be the reason for its inactivation.

Several types of antiviral agents other than Ag nanoparticles have now been widely studied, and their antiviral mechanisms differ depending on the type of agent $[5,13]$. Therefore, it is possible that inactivation of antiviral agent might be caused by components of the virus suspension, as well as by other experimental conditions.

\section{Conclusion}

A quantitative method for antiviral activity testing of textile fabrics was investigated. We found that virus infectivity titers were significantly affected by such experimental conditions as the concentration of the medium of the virus suspension, the contact temperature, and the type of textile fabric. To estimate the antiviral efficiency under stable conditions for viruses, a lower temperature $\left(4^{\circ} \mathrm{C}\right)$ and a lower EMEM concentration (1/10 EMEM) appear to be appropriate. We also showed that the viruses can be deactivated by contact with textile fabrics, even in the absence of antiviral agents. Therefore, a relative comparison with untreated specimen is essential in testing of treated samples. Furthermore, we also suggest that components present in the test medium might suppress the activity of antiviral agents. To summarize, it is necessary to choose appropriate experimental conditions for each particular testing purpose, taking into account the possibilities of virus inactivation and/or antiviral agent deactivation under the experimental conditions.

\section{Acknowledgements}

We would like to thank emeritus professor Hiroki Kourai for his guidance and many helpful suggestions. We also thank Daijiro Kitagawa, Yoshiki Kubo, and Tomoya Kosaka (Osaka University) for their technical support. This research was partially supported by a Grant-in-Aid for Scientific Research (15H02342) from the Ministry of Education, Culture, Sports, Science and Technology of Japan.

\section{References}

1. Lee HJ, Yeo SY, Jeong SH (2003) Antibacterial effect of nanosized silver colloidal solution on textile fabrics. J Mater Sci 38: 2199-2204.

2. Imoto Y, Seino S, Nakagawa T, Yamamoto TA (2017) Comparison of quantitative antifungal testing methods for textile fabrics. Biocontrol Sci 22: 47-53.

3. No HK, Park NY, Lee SH, Meyers SP (2002) Antibacterial activity of chitosans and chitosan oligomers with different molecular weights. Int $\mathrm{J}$ Food Microbiol 74: 65-72.

4. Johnson LB, Kauffman CA (2003) Voriconazole: A new triazole antifungal agent. Clin Infect Dis 36: 630-637.

5. De Clercq E (2006) Antiviral agents active against influenza A viruses. Nat Rev Drug Discov 5: 1015-1025.

6. Takizawa T, Matsukawa S, Higuchi Y, Nakamura S, Nakanishi Y, et al (1993) Induction of programmed cell death (apoptosis) by influenza virus infection in tissue cells. J Gen Virol 74: 2347-2355.

7. Furuta $Y$, Takahashi K, Kuno-Maekawa M, Sangawa H, Uehara S, et al. (2005) Mechanism of action of T-705 against influenza virus. Antimicrob Agents Chemother 49: 981-986.

8. Seino S, Imoto Y, Kitagawa D, Kubo Y, Kosaka T, et al. (2015) Radiochemical synthesis of silver nanoparticles onto textile fabrics and their antibacterial activities. J Nucl Sci Technol 53: 1021-1027.

9. International Organization for Standardization (2014) ISO 18184: Textiles-Determination of antiviral activity of textile products. ISO, Geneva.

10. Sondi I, Salopek-Sondi B (2004) Silver nanoparticles as antimicrobial agent: A case study on E. coli as a model for Gram-negative bacteria. J Colloid Interface Sci 275: 177-182.

11. Fraise AP, Lambert PA, Maillard JY (2008) Russell, Hugo \& Ayliffe's Principles and Practice of Disinfection, Preservation \& Sterilization, (4th edn), Blackwell, Malden MA.

12. Liu J, Sonshine DA, Shervani S, Hurt RH (2010) Controlled release of biologically active silver from nanosilver surfaces. ACS Nano 4: 6903-6913.

13. Borkow G, Gabbay J (2004) Putting copper into action: Copperimpregnated products with potent biocidal activities. FASEB J 18: 1728-1730. 